\title{
A NOTE ON THE ERGODIC THEOREM
}

\author{
TAKESHI YOSHIMOTO
}

\begin{abstract}
Let $M$ be a positive regular shift-invariant method of summability and let $T$ be a one-to-one transformation which maps $X$ onto $X$ and which is $\mathscr{B}$-bimeasurable, i.e., $A \in \mathscr{B}$ if and only if $T A \in \mathscr{B}$, where $(X, \mathscr{B})$ is a measurable space. Then it is proved that if for a finite measure $\mu$ on $\mathscr{B}$ the sequence $\left\{\mu\left(T^{m} A\right)\right\}$ is $M$-summable for each $A \in \mathcal{B}$, then for any real $r \geqslant 1$ the sequence $\left\{f \circ T^{m}\right\}$ is $(C, r)$-summable $\mu$-almost everywhere for every bounded $\mathscr{B}$-measurable function $f$ defined on $X$. The result includes the Blum-Hanson theorem.
\end{abstract}

Let $X$ be a nonempty set, $\mathscr{B}$ a $\sigma$-algebra of subsets of $X$, and $Y$ a reflexive Banach space with norm denoted by $\|\cdot\| \|$. The symbol $L(X: Y)$ will stand for the class of all bounded strongly $\Re$-measurable $Y$-valued functions defined on $X$. Let $M=\left(m_{i j}\right)$ $(i, j=0,1,2, \ldots)$ be an infinite matrix satisfying:

(i) $m_{i j} \geqslant 0$ for all $i$ and $j$,

(ii) $\sum_{j=0}^{\infty} m_{i j} \leqslant C<\infty$ uniformly in $i$,

(iii) $\lim _{i \rightarrow \infty} m_{i j}=0$ for all $j$, and

(iv) $\lim _{i \rightarrow \infty} \sum_{j=0}^{\infty} m_{i j}=1$.

Such a matrix $M$ defines a positive regular method of summability and a good deal is known about such methods. The method $M$ is called shift-invariant provided for every sequence $\left\{u_{n}\right\}$ of real numbers for which $\lim _{i \rightarrow \infty} \sum_{j=0}^{\infty} m_{i j} u_{j}=u$ exists and is finite we have $\lim _{i \rightarrow \infty} \sum_{j=0}^{\infty} m_{i j} u_{j+1}=u$. Fix a real $r \geqslant 1$ arbitrarily and put $A_{0}^{(r)}=1$ and $A_{n}^{(r)}=(r+1)(r+2) \cdots(r+n) / n$ ! for $n=1,2,3, \ldots$ The $(C, r)$-method, i.e., $m_{i j}^{(r)}=A_{i-j}^{(r-1)} / A_{i}^{(r)}$ for $j=0,1, \ldots, i$ and $m_{i j}^{(r)}=0$ for $j=i+1, i+2, \ldots$, is an example of a positive regular shift-invariant method of summability. A sequence $\left\{u_{n}\right\}$ is called $M$-summable if $\lim _{i \rightarrow \infty} \sum_{j=0}^{\infty} m_{i j} u_{j}$ exists. We now prove the following $(C, r)$-generalization of the Blum-Hanson theorem [1].

THEOREM 1. Let $\mu$ be a finite measure defined on $\Re, T$ a one-to-one $\Re$-bimeasurable transformation on $X$, and $M$ a positive regular shift-invariant method of summability. If $\left\{\mu\left(T^{m} A\right)\right\}$ is $M$-summable for each $A \in \Re$ then the sequence $\left\{f \circ T^{m}\right\}$ is $(C, r)$-summable (strongly in $Y$ ) $\mu$-almost everywhere for every $f \in L(X: Y)$, i.e., there exists a function $f^{*} \in L(X: Y)$ such that

$$
f^{*}(x)=\lim _{n \rightarrow \infty} \sum_{m=0}^{n}\left(A_{n-m}^{(r)} / A_{n}^{(r)}\right) f\left(T^{m} x\right),
$$

Received by the editors May 5, 1983.

1980 Mathematics Subject Classification. Primary 47A35; Secondary 28 C05.

$K e y$ words and phrases. Regular summation method, $M$-summable, $(C, r)$-summable, Blum-Hanson theorem.

(C1984 American Mathematical Society $0002-9939 / 84 \$ 1.00+\$ .25$ per page 


$$
f^{*}(T x)=f^{*}(x)
$$

(strongly in $Y$ ) for $\mu$-almost all $x \in X$.

In what follows, the symbol $U_{T}$ will be used for denoting the induced operator of $T$, i.e., $U_{T} f=f \circ T$. We note that the class of finite measures for which our theorem holds includes the class of finite $T$-invariant measures. In particular, if one takes $r=1$ and $Y$ to be the linear space of real or complex numbers, then our result becomes the Blum-Hanson theorem. The operator-theoretical treatment of $(C, r)$ summability, including the $\sigma$-finite case of invariant measures, is given in [4].

Proof of Theorem 1. Let $\lambda(A)=\lim _{i \rightarrow \infty} \lambda_{i}(A)$ for each $A \in \mathscr{R}$, where $\lambda_{i}(A)=$ $\sum_{j=0}^{\infty} m_{i j} \mu\left(T^{j} A\right)(i=0,1,2, \ldots)$. Then $\lambda$ is a finite measure on $\mathscr{B}$ which is invariant under $T$. Indeed this follows from the shift-invariance of $M$ and the fact that if $\lambda_{n}$ is a sequence of finite signed measures such that $\lambda(A)=\lim _{n \rightarrow \infty} \lambda_{n}(A)$ exists and is finite for every $A \in \mathscr{B}$, then $\lambda$ is a signed measure (see, for example, Halmos [3, p. 170]). Clearly, $\mu$ is absolutely continuous with respect to $\lambda$, i.e., $\lambda(A)=0, A \in \mathscr{B}$ implies $\mu(A)=0$. Since $L_{2}(X, \mathscr{B}, \lambda, Y)$ is reflexive, it follows that by the YosidaKakutani mean ergodic theorem, the functions $f$ of the form $f=g+\left(I-U_{T}\right) h$, $g=U_{T} g, g \in L_{2}(X, \Re, \lambda, Y), h \in L_{\infty}(X, \mathscr{B}, \lambda, Y)$, are dense in $L_{2}(X, \Re, \lambda, Y)$. For such a function $f$, one gets

$$
\begin{aligned}
\sum_{m=0}^{n} \frac{A_{n-m}^{(r-1)}}{A_{n}^{(r)}} U_{T}^{m} f(x)= & \left(\sum_{m=0}^{n} \frac{A_{n-m}^{(r-1)}}{A_{n}^{(r)}}\right) g(x)+\frac{A_{n}^{(r-1)}}{A_{n}^{(r)}} h(x)-\frac{A_{0}^{(r-1)}}{A_{n}^{(r)}} U_{T}^{n+1} h(x) \\
& +\sum_{m=1}^{n}\left[\frac{A_{n-m}^{(r-1)}-A_{n-m+1}^{(r-1)}}{A_{n}^{(r)}}\right] U_{T}^{m} h(x) .
\end{aligned}
$$

Note that $\sum_{m=0}^{n} A_{n-m}^{(r-1)}=A_{n}^{(r)}$ and $\lim _{n \rightarrow \infty} A_{n}^{(r)}=\infty$. It follows that

$$
\lim _{n \rightarrow \infty} \sum_{m=0}^{n} \frac{A_{n-m}^{(r-1)}}{A_{n}^{(r)}} g(x)=g(x), \quad \lim _{n \rightarrow \infty} \frac{A_{n}^{(r-1)}}{A_{n}^{(r)}} h(x)=0
$$

and

$$
\lim _{n \rightarrow \infty} \frac{A_{0}^{(r-1)}}{A_{n}^{(r)}} U_{T}^{n+1} h(x)=0
$$

strongly in $Y$ for $\lambda$-almost all $x \in X$. Furthermore, for any integer $p>1$,

$$
\begin{array}{r}
\left\|\left|\sum _ { m = 1 } ^ { n } [ \frac { A _ { n - m } ^ { ( r - 1 ) } - A _ { n - m + 1 } ^ { ( r - 1 ) } } { A _ { n } ^ { ( r ) } } ] U _ { T } ^ { m } h ( x ) \left\|\left|\leqslant\|h\|_{\infty} \sum_{m=1}^{\infty}\right| \frac{A_{n-m}^{(r-1)}-A_{n-m+1}^{(r-1)}}{A_{n}^{(r)}} \mid\right.\right.\right. \\
\leqslant\|h\|_{\infty}\left(\sum_{m=1}^{p-1}\left|\frac{A_{n-m}^{(r-1)}-A_{n-m+1}^{(r-1)}}{A_{n}^{(r)}}\right|+\sum_{m=p}^{\infty}\left|\frac{A_{n-m}^{(r-1)}-A_{n-m+1}^{(r-1)}}{A_{n}^{(r)}}\right|\right) .
\end{array}
$$

Now the Cesàro matrix $\left(m_{i j}^{(r)}\right)$ satisfies the uniformity condition in the sense of Cohen:

$$
\lim _{p \rightarrow \infty} \sum_{j=p}^{\infty}\left|m_{i, j+1}^{(r)}-m_{i j}^{(r)}\right|=0
$$


uniformly in $i$ (see Cohen [2]). So, given $\varepsilon>0$, there is a number $n(\varepsilon)$ such that

$$
\left\|\sum_{m=0}^{n}\left[\frac{A_{n-m}^{(r-1)}-A_{n-m+1}^{(r-1)}}{A_{n}^{(r)}}\right] U_{T}^{m} h(x)\right\| \leqslant 2\|h\|_{\infty} \varepsilon, \quad n>n(\varepsilon) .
$$

Thus the limit, $\lim _{n \rightarrow \infty} \sum_{m=0}^{n}\left(A_{n-m}^{(r-1)} / A_{n}^{(r)}\right) U_{T}^{m} f$, exists (in $Y$ ) $\lambda$-almost everywhere for every $f$ in a set dense in $L_{2}(X, \mathscr{T}, \lambda, Y)$. However,

$$
\sup _{n \geqslant 0}\left\|\left|\sum_{m=0}^{n} \frac{A_{n-m}^{(r-1)}}{A_{n}^{(r)}} U_{T}^{m} f\right|\right\| \leqslant r \sup _{n \geqslant 0}\left\|\left|\frac{1}{n+1} \sum_{m=0}^{n} U_{T}^{m} f \|\right|<\infty\right.
$$

$\lambda$-almost everywhere for any $f \in L_{2}(X, \Re, \lambda, Y)$. Therefore by the Banach convergence theorem, $\lim _{n \rightarrow \infty} \sum_{m=0}^{n}\left(A_{n-m}^{(r-1)} / A_{n}^{(r)}\right) U_{T}^{m} f$ exists (in $Y$ ) $\lambda$-almost everywhere for every $f \in L_{2}(X, \Re, \lambda, Y)$. Since $L(X: Y) \subset L_{2}(X, \Re, \lambda, Y)$ by the finiteness of $\lambda$, Theorem 1(a) follows at once from this by the absolute continuity of $\mu$ with respect to $\lambda$. To see Theorem l(b) it suffices to note that given $\varepsilon>0$ there is a number $n(\varepsilon)$ such that, for $f \in L(X: Y)$,

$$
\begin{aligned}
& \|\| \sum_{m=0}^{n} \frac{A_{n-m}^{(r-1)}}{A_{n}^{(r)}} U_{T}^{m} f-\sum_{m=0}^{n} \frac{A_{n-m}^{(r-1)}}{A_{n}^{(r)}} U_{T}^{m+1} f \| \mid \\
& \quad \leqslant\left\|\left|\frac{A_{n}^{(r-1)}}{A_{n}^{(r)}} f\|+\|\right|\right\| \frac{A_{0}^{(r-1)}}{A_{n}^{(r)}} U_{T}^{n+1} f\|+\| \sum_{m=1}^{n}\left[\frac{A_{n-m}^{(r-1)}-A_{n-m+1}^{(r-1)}}{A_{n}^{(r)}}\right] U_{T}^{m} f\|\| \\
& \quad<4\|f\|_{\infty} \varepsilon, \quad n>n(\varepsilon) .
\end{aligned}
$$

Hence Theorem 1 has been proved.

The following corollaries are immediate from Theorem 1 using the Lebesgue bounded convergence theorem.

Corollary 1 (CF. [1, Corollary 2]). On the hypothesis of Theorem 1, if the sequence $\left\{\mu\left(T^{m} A\right)\right\}$ is $M$-summable for each $A \in \Re$ then it is also $(C, r)$-summable.

Corollary 2 (CF. [1, CoRollary 1]). Let $T$ be a one-to-one 乃-bimeasurable transformation on $X$ and let $\mu$ be a finite measure on $\Re$. Then the following statements are equivalent:

(1) the sequence $\left\{\mu\left(T^{m} A\right)\right\}$ is $(C, 1)$-summable for each $A \in \mathscr{B}$,

(2) the sequence $\left\{\mu\left(T^{m} A\right)\right\}$ is $(C, r)$-summable for each $A \in \Re$,

(3) the sequence $\left\{U_{T}^{m} f\right\}$ is $(C, 1)$-summable $\mu$-almost everywhere for each $f \in$ $L(X: Y)$,

(4) the sequence $\left\{U_{T}^{m} f\right\}$ is $(C, r)$-summable $\mu$-almost everywhere for each $f \in$ $L(X: Y)$.

More generally, we consider a regular summation method $A=\left(a_{n m}: n, m=\right.$ $0,1,2, \ldots)$. Such methods are characterized by the familiar Silverman-Toeplitz conditions (ii)-(iv) mentioned above. Modifying the argument used in the proof of 
Theorem 1 (cf. [4]) we have

THEOREM 2. Let $A=\left(a_{n m}\right)$ be a regular summation method satisfying the uniformity condition in the sense of Cohen. Let $T$ be a $B$-measurable point transformation on $X$ and $\mu$ a $\sigma$-finite measure on $\Re$ such that $U_{T}$ is contracting on $L_{1}(X, \Re, \mu, Y)$ as well as on $L_{\infty}(X, \Re, \mu, Y)$. Assume there is a positive constant $C$ such that for any $f \in$ $L_{p}(X, \Re, \mu, Y), 1 \leqslant p<\infty$,

$$
\sup _{n}\|\| \sum_{m=0}^{\infty} a_{n m} U_{T}^{m} f\left\|\left|\leqslant C \sup _{n}\left\|\left|\frac{1}{n+1} \sum_{m=0}^{n} U_{T}^{m} f \|\right|\right.\right.\right.
$$

$\mu$-almost everywhere. Then the sequence $\left\{U_{T}^{m} f\right\}$ is A-summable (strongly in $Y$ ) $\mu$-almost everywhere for each $f \in L_{p}(X, \Re, \mu, Y), 1 \leqslant p<\infty$.

\section{REFERENCES}

1. J. R. Blum and D. L. Hanson, A note on the ergodic theorem, Proc. Amer. Math. Soc. 16 (1965), 413-414.

2. L. W. Cohen, On the mean ergodic theorem, Ann. of Math. (2) 41 (1940), 505-509.

3. P. R. Halmos, Measure theory, Van Nostrand, New York, 1950.

4. T. Yoshimoto, Generalized ergodic inequalities and ergodic theorems, Probability Theory and Mathematical Statistics (ed. by K. Ito and J. V. Prokhorov): Proceedings of the 4th USSR-Japan Symposium, held at Tbilisi, USSR. August 23-29, 1982, Lectures Notes in Math., vol. 1021, Springer-Verlag (to appear).

Department of Mathematics, Toyo University, Saitama, Japan 